\title{
Strong-field tests of gravity using pulsars \& black holes
}

\author{
Michael Kramer* \\ $J B C A /$ University of Manchester \\ E-mail: Michael.Kramer@manchester.ac.uk
}

Ever since their discovery, pulsars have been used as precise cosmic clocks. Their observations in binary systems takes us beyond the weak-field regime of the solar-system in the study of theories of gravity. Their contribution is crucial as no test can be considered to be complete without probing the strong-field realm of gravitational physics by finding and timing pulsars. This is particularly highlighted by the discovery of the first double pulsar system which is unique in that both neutron stars are detectable as radio pulsars. This, combined with significantly higher mean orbital velocities and accelerations when compared to other binary pulsars, provides the best available testbed for general relativity and alternative theories of gravity in the strong-field regime. However, more extreme binary systems are expected to exist, and we expect them to be uncovered in sensitive surveys with the Square Kilometre Array (SKA). The SKA will be a radio telescope with a collecting area that will exceed that of existing telescopes by a factor of a hundred or so. This sensitivity allows us to perform a Galactic Census of pulsars which will discover a large fraction of active pulsars beamed to us, including the long-sought for pulsar-black hole systems. Regular observations of these systems with the SKA will probe the ultra-strong field limit of relativistic gravity. By using pulsar timing we can determine the properties of stellar and massive black holes, thereby testing the Cosmic Censorship Conjecture and the No-Hair theorem. The large number of millisecond pulsars discovered with the SKA will also provide us with a dense array of precision clocks on the sky. These clocks will act as the multiple arms of a huge gravitational wave detector, which can be used to detect and measure the stochastic cosmological gravitational wave background that is expected from a number of sources.

From Planets to Dark Energy: the Modern Radio Universe

October 1-5 2007

The University of Manchester, UK

\footnotetext{
* Speaker.
} 


\section{Introduction}

Almost a hundred years after Einstein formulated his theory of general relativity (GR), efforts in testing GR and its concepts are still being made by many colleagues around the world, using many different approaches. To date GR has passed all experimental and observational tests with flying colours, but in particular in light of recent progress in observational cosmology, presented also at this conference, the question of whether alternative theories of gravity need to be considered is as topical as ever.

Many experiments are designed to achieve ever more stringent tests by either increasing the precision of the tests, or by testing different, new aspects. Some of the most stringent tests are obtained by satellite experiments in the solar system, providing exciting limits on the validity of GR and alternative theories of gravity like tensor-scalar theories. However, solar-system experiments are made in the gravitational weak-field regime, while deviations from GR may appear only in strong gravitational fields. It happens that nature provides us with an almost perfect laboratory to test the strong-field regime - radio pulsars.

Pulsars are highly magnetized rotating neutron stars and are unique and versatile objects which can be used to study an extremely wide range of physical and astrophysical problems. Besides testing theories of gravity one can study the Galaxy and the interstellar medium, stars, binary systems and their evolution, plasma physics and solid state physics under extreme conditions. For tests of theories of gravity, pulsars are in particular useful if they are in orbit with another compact body. In such case, we encounter two gravitational test masses of which (at least) one is fitted with a precise cosmic clock. By tracing the motion of this clock in the curved space-time of its companion, we can test the predictions of GR and alternative theories of gravity.

This type of experiment is exemplified by the first ever discovered double pulsar [1, 2]. This unique system allows us to test many aspects of gravitational theories at the same time, representing a truly unique laboratory for relativistic gravity. Despite the amazing properties of the Double Pulsar discussed later, it is by far not the most extreme system that we can expect to find. Indeed, more exciting systems should exist in the Galaxy, of which the crown jewel would undoubtedly be a pulsar orbiting a black hole. Such a system would be a unique probe for testing gravity, allowing us to determine and precisely measure the properties of black holes. However, a pulsar - black hole system is certainly rare, and even if we were to find it today, current technology would not be sufficient to perform the necessary experiments. But pulsar astronomers do not despair since the solution is already on the drawing board: it is called the Square-Kilometre Array (SKA)! The SKA will provide an unique gigantic collecting area which will not only allow to us to discover the whole potentially observable Galactic pulsar population, including pulsar - black hole systems, but it also also provides us with the sensitivity to achieve the needed timing precision to exploit the discovered systems. We will outline these experiments further below.

\section{Binary pulsars as gravitational laboratories}

Strictly speaking, the separation between a binary pulsar and its companion is large enough for the pulsar to move in the weak gravitational field of a companion. However, they do provide precision tests of the strong-field regime. This becomes clear when considering strong gravitational 
self-field effects which are predicted by the majority of alternative theories. Such effects would, for instance, clearly affect the pulsars' orbital motion, allowing us to search for these effects and hence providing us with a unique precision strong-field test of gravity.

The bodies encountered in the solar system are not massive and compact enough to allow similar experiments, so that the strong-field limit which is largely unexplored. Tests involving the observations of X-ray binaries may help, but the interpretation of these results depends to some extent on physics (e.g. accretion physics) which is complex so that the involved conditions are known with only limited precision. In contrast, binary pulsars with white dwarf and neutron star companions represent systems of test masses that only interact gravitationally, so that their motions should be fully explained by the theory of gravity to be tested. The best test bed known is the unique Double Pulsar system.

\section{The Double Pulsar}

Our team discovered the 22.8-ms pulsar J0737-3039 in April 2003 [1] in an extension to the hugely successful Parkes Multi-beam survey [3]. It was soon found to be a member of the most extreme relativistic binary system ever discovered: its short orbital period $\left(P_{\mathrm{b}}=2.4 \mathrm{hrs}\right)$

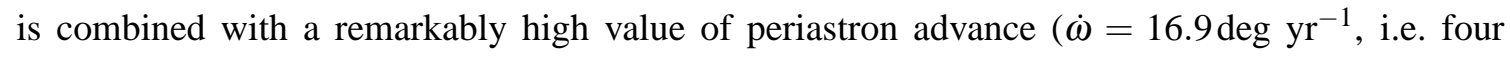
times larger than for the Hulse-Taylor pulsar PSR B1913+16). This large precession of the orbit was measurable after only a few days of observations. The system parameters predict that the two members of the binary system will coalesce on a short time scale of only $\sim 85 \mathrm{Myr}$. This boosts the hopes for detecting a merger of two neutron stars with first-generation ground-based gravitational wave detectors by a factor of 5 to 10 compared to previous estimates based on only the double neutron stars B1534+12 and B1913+16 [1], 印.

In October 2003, we detected radio pulses from the second neutron star [2]. The reason why signals from the 2.8-s pulsar companion (now called PSR J0737-3039B, hereafter "B") to the millisecond pulsar (now called PSR J0737-3039A, hereafter "A") had not been found earlier, became clear when it was realized that B was only bright for two short parts of the orbit. For the remainder of the orbit, the pulsar B is extremely weak and only detectable with the most sensitive equipment. The detection of a young companion B around an old millisecond pulsar A confirms the evolution scenario proposed for recycled pulsars and provides a truly unique laboratory for relativistic gravity.

Timing observations of PSR J0737-3039A/B have been undertaken using the 64-m Parkes radio telescope in New South Wales, the 76-m Lovell radio telescope at Jodrell Bank Observatory, UK, and the 100-m Green Bank Telescope in West Virginia. Because of its narrower and more stable pulse profile, pulsar A can be timed with a much higher precision than B. The latest timing results [5] using the DDS timing model [6] resulted in the measurement of five "Post-Keplerian" (PK) parameters. The PK parameters are "corrections" that need to applied to a simple Keplerian orbit in order to describe the observed pulse times-of-arrival (TOAs). It is important to note that the PK parameters are measured as additional parameters in a theory independent and phenomenological way, so that they can be compared with predictions from gravitational theories. One can show [7] that for point masses with negligible spin contributions, the PK parameters should only be functions of the a priori unknown pulsar and companion mass and the easily measurable Keplerian 
parameters. The actual functions will be different for different theories of gravity, but with the two masses as the only free parameters, an observation of two PK parameters will already determine the masses uniquely in the framework of the given theory. The measurement of a third or more PK parameters then provides a consistency check for the theory to be tested.

The first PK parameter to be measured for the Double Pulsar was $\dot{\omega}$, describing a relativistic advance of periastron. It provides an immediate measurement of the total mass of the system, $\left(M_{A}+M_{B}\right)$. The second PK parameter $\gamma$ denotes the amplitude of delays in arrival times caused by the varying effects of the gravitational redshift and time dilation (second order Doppler) as the pulsar moves in its elliptical orbit at varying distances from the companion and with varying speeds.

Two other PK parameters, $r$ and $s$, are related to the Shapiro delay caused by the gravitational field of the companion. It describes the extra pathlength caused by the curvature of space-time to be travelled by the electromagnetic signal when it passes the companion. Usually, depending on timing precision, a Shapiro delay can only be measured if the orbit is seen nearly edge-on. However, the observation of short eclipses in the emission of A during superior conjunction indicate that we are observing the Double Pulsar almost completely edge-on. Using GR, we can identify the measured parameter $s$ with $\sin i$ where $i$ is the inclination angle of the orbit. Indeed, the value of $s$ derived from our timing observations corresponds to $i=88^{\circ} .69_{-0^{\circ} .76}^{+0^{\circ} .}$

After less than three years since the Double Pulsar's discovery, we also measured a decay of the orbit due to gravitational wave damping which is expressed by a change in orbital period, $\dot{P}_{\mathrm{b}}$. The value of $\dot{P}_{\mathrm{b}}$ corresponds to a shrinkage of the orbit at a rate of $7 \mathrm{~mm}$ per day.

In addition to tests enabled by the PK parameters, the access to the orbit of both neutron stars - by timing A and $\mathrm{B}$ - provides yet another constraint on gravitational theories that is qualitatively different from what has been possible with previously known double neutron stars: using Kepler's third law, the measurement of the projected semi-major axes of both orbits yields the mass ratio,

$$
R\left(M_{A}, M_{B}\right) \equiv M_{A} / M_{B}=x_{B} / x_{A} .
$$

For every realistic theory of gravity, we can expect $R$ to follow this simple relation [7], at least to $1 \mathrm{PN}$ order. Most importantly, the $R$ value is not only theory-independent, but also independent of strong-field (self-field) effects which is not the case for the PK parameters. In other words, any combination of masses derived from the PK parameters must be consistent with the mass ratio. The ability to measure this quantity provides therefore an important and unique constraint. With five PK parameters already available, this additional constraint also makes the double pulsar the most overdetermined system to date where the most relativistic effects can be studied in the strong-field limit.

One can display these tests elegantly in a "mass-mass" diagram as shown in Figure 11. Measurement of the PK parameters gives curves on this diagram that are in general different for different theories of gravity but which should intersect in a single point, i.e., at a pair of mass values, if the theory is valid [7]. Together with the mass ratio $R$, the PK parameters provide a total of six curves in the mass-mass diagram. Determining an intersection point using a pair of curves, we obtain four independent tests of GR, more than for any other known system.

Figure 11 shows that all measured constraints are consistent with GR. The most precisely measured PK parameter currently available is the precession of the longitude of periastron, $\dot{\omega}$. We can 
Table 1: Parameters for PSR J0737-3039A (A) and PSR J0737-3039B (B) as measured by [5]. The five Keplerian binary parameters $\left(P_{b}, e, \omega, T_{0}\right.$, and $\left.x\right)$ are derived for pulsar A. The first four of these (with an offset of $180^{\circ}$ added to $\omega$ ) and the position parameters were assumed when fitting for B's parameters. Five post-Keplerian parameters have been measured so far. An independent fit of $\dot{\omega}$ for B yielded a value (shown in square brackets) that is consistent with the much more precise result for A. The value derived for A was adopted in the final analysis.

\begin{tabular}{|c|c|c|}
\hline Timing parameter & PSR J0737-3039A & PSR J0737-3039B \\
\hline Right Ascension $\alpha$ & $07^{\mathrm{h}} 37^{\mathrm{m}} 51^{\mathrm{s}} .24927(3)$ & - \\
\hline Declination $\delta$ & $-30^{\circ} 39^{\prime} 40^{\prime \prime} .7195(5)$ & - \\
\hline Proper motion in the RA direction ( mas $\mathrm{yr}^{-1}$ ) & $-3.3(4)$ & - \\
\hline Proper motion in Declination (mas $\mathrm{yr}^{-1}$ ) & $2.6(5)$ & - \\
\hline Parallax, $\pi$ (mas) & $3(2)$ & - \\
\hline Spin frequency $v(\mathrm{~Hz})$ & $44.054069392744(2)$ & $0.36056035506(1)$ \\
\hline Spin frequency derivative $\dot{v}\left(\mathrm{~s}^{-2}\right)$ & $-3.4156(1) \times 10^{-15}$ & $-0.116(1) \times 10^{-15}$ \\
\hline Timing Epoch (MJD) & 53156.0 & 53156.0 \\
\hline Dispersion measure DM $\left(\mathrm{cm}^{-3} \mathrm{pc}\right)$ & $48.920(5)$ & - \\
\hline Orbital period $P_{b}$ (day) & $0.10225156248(5)$ & - \\
\hline Eccentricity $e$ & $0.0877775(9)$ & - \\
\hline Projected semi-major axis $x=(a / c) \sin i(\mathrm{~s})$ & $1.415032(1)$ & $1.5161(16)$ \\
\hline Longitude of periastron $\omega(\mathrm{deg})$ & $87.0331(8)$ & $87.0331+180.0$ \\
\hline Epoch of periastron $T_{0}$ (MJD) & $53155.9074280(2)$ & - \\
\hline Advance of periastron $\dot{\omega}(\mathrm{deg} / \mathrm{yr})$ & $16.89947(68)$ & {$[16.96(5)]$} \\
\hline Gravitational redshift parameter $\gamma(\mathrm{ms})$ & $0.3856(26)$ & - \\
\hline Shapiro delay parameter $s$ & $0.99974(-39,+16)$ & - \\
\hline Shapiro delay parameter $r(\mu \mathrm{s})$ & $6.21(33)$ & - \\
\hline Orbital period derivative $\dot{P}_{b}$ & $-1.252(17) \times 10^{-12}$ & - \\
\hline Timing data span (MJD) & $52760-53736$ & $52760-53736$ \\
\hline RMS timing residual $\sigma(\mu \mathrm{sec})$ & 54 & 2169 \\
\hline Orbital inclination angle (deg) & \multicolumn{2}{|c|}{$88.69(-76,+50)$} \\
\hline Mass function $\left(M_{\odot}\right)$ & $0.29096571(87)$ & $0.3579(11)$ \\
\hline Mass ratio, $R$ & \multicolumn{2}{|c|}{$1.0714(11)$} \\
\hline Total system mass $\left(M_{\odot}\right)$ & \multicolumn{2}{|c|}{$2.58708(16)$} \\
\hline Neutron star mass $\left(m_{\odot}\right)$ & $1.3381(7)$ & $1.2489(7)$ \\
\hline
\end{tabular}

combine this with the theory-independent mass ratio $R$ to derive the masses given by the intersection region of their curves: $m_{\mathrm{A}}=1.3381 \pm 0.0007 \mathrm{M}_{\odot}$ and $m_{\mathrm{B}}=1.2489 \pm 0.0007 \mathrm{M}_{\odot}$. Assuming GR and using these masses and the Keplerian parameters, we can predict values for the remaining PK parameters. Four independent tests that are currently available. The Shapiro delay gives the most precise test, with $s_{\mathrm{obs}} / s_{\text {pred }}=0.99987 \pm 0.00050$. This is by far the best test of GR in the strong-field limit, having a higher precision than the test based on the observed orbit decay in the PSR B1913+16 system with a 30-year data span [8]. As for the PSR B1534+12 system [9], the PSR J0737-3039A/B Shapiro-delay test is complementary to that of B1913+16 since it is not based on predictions relating to emission of gravitational radiation from the system [10]. Most importantly, all four independent tests of GR provided by the Double Pulsar are qualitatively different from all previous tests because they include one constraint $(R)$ that is independent of the assumed theory of gravity at the 1PN order. As a result, for any theory of gravity, the intersection point is expected to lie on the mass ratio line in Figure 11. GR also passes this additional constraint with the best precision so far. 


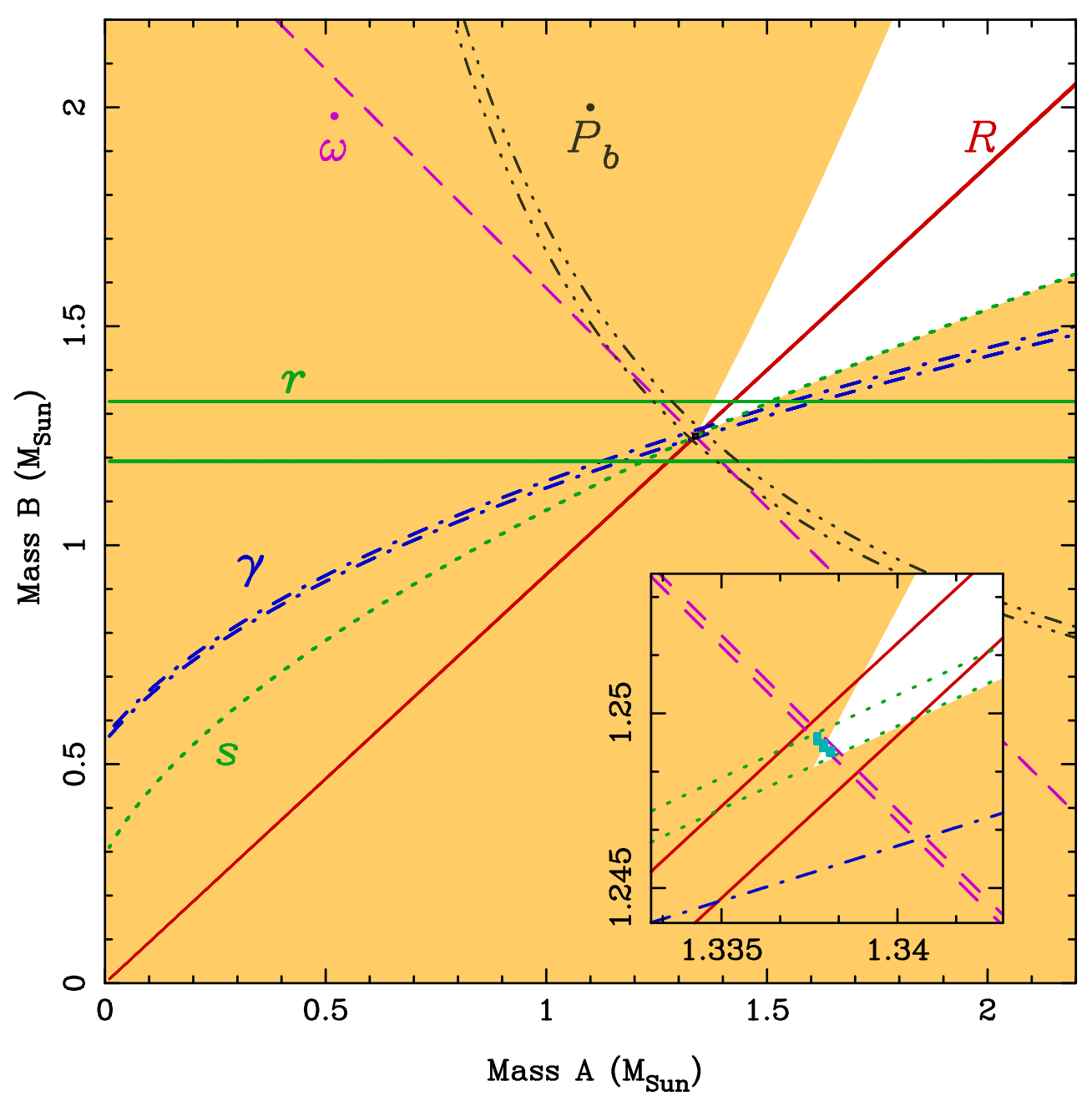

Figure 1: 'Mass-mass' diagram showing the observational constraints on the masses of the neutron stars in the double pulsar system J0737-3039 [5]. The shaded regions are those that are excluded by the Keplerian mass functions of the two pulsars. Further constraints are shown as pairs of lines enclosing permitted regions as given by the observed mass ratio and PK parameters as predicted by general relativity. Inset is an enlarged view of the small square encompassing the intersection of these constraints (see text).

\section{Alternative theories}

Alternative theories of gravity may not only deviate from GR in predicting different values for PK parameters but they may also involve effects that are completely absent in GR. Some theories, for instance, predict that the Universe's global matter distribution selects a preferred rest frame for local gravitational physics, so that in contrast to GR, the outcome of gravitational experiments depends on the motion of the laboratory with respect to this preferred frame. In particular, theories in which gravity is partially mediated by a vector field or a second tensor field are known to exhibit such preferred-frame effects (PFEs) whose strength is determined by cosmological matching parameters. As Wex \& Kramer [11] recently demonstrated, such PFEs are also testable with binary pulsars. In particular, they present a consistent, theory-independent methodology to measure PFEs 
in binary pulsars that exhibit a high rate of periastron advance. They show that the existence of a preferred frame for gravity would lead to characteristic periodic changes in the orbital parameters, with a period determined by the timescale for the orbital precession.

Based on the work by Damour \& Deruelle [12, 13, Wex \& Kramer developed a timing model that describes orbital variations due to PFEs and demonstrate how timing observations can be used to either measure or constrain the parameters related to a violation of the local Lorentz invariance of gravity in the strong internal fields of neutron stars. In particular, Wex \& Kramer point out that for the case that PFEs should indeed exist, two pairs of their newly introduced timing parameters will have a unique relationship that depends only on the orbital parameters of the binary system. This has far reaching consequences: Using the system parameters of the Double Pulsar, they compute the two numbers that we should expect to measure in the presence of PFEs. If our observed values deviate from these two numbers, the existence of preferred frames and a violation of gravitational Lorentz invariance can be ruled out.

As Wex \& Kramer [1] point out, the Double Pulsar is indeed particularly suitable for testing PFEs. As the characteristic PFE signatures are periodic with the orbital precession period, periastron has to have advanced sufficiently far to separate the PFE timing amplitudes from other orbital parameters. However, at the moment, even with the huge periastron advance of the Double Pulsar, the current data span is yet too short, so that only an upper limits for PFEs can be derived. However, with longer time-spans, and more coverage of the periastron angle, a more constraining fit for PFE effects will be possible. We note that while the current limits are not very competitive with corresponding weak-field tests in the solar system, the Double Pulsar limits are qualitatively different as they probe strong-field effects that do not occur in the solar system.

While the Double Pulsar will not only allow us to detect a preferred frame should it exist, it will also constrain its direction. But this test is not equally sensitive to all directions, but depends on the orbital orientation relative to the preferred frame. For this reason, it is useful to combine the data of several binary pulsars, of which many will be discovered with a future telescope known as the SKA.

\section{The Square-Kilometre-Array (SKA)}

Through its sensitivity, sky and frequency coverage, the SKA will discover a very large fraction of the pulsars in the Galaxy, resulting in about 20,000 to 30,000 pulsars [14, 15]. This number represents essentially all active pulsars that are beamed toward Earth and includes the discovery of more than 1,000 millisecond pulsars (MSPs). This impressive yield effectively samples every possible outcome of the evolution of massive binary stars. The sensitivity of the SKA allows much shorter integration times, so that searches for compact binary pulsars will no longer be limited. Among the discovered sources, pulsar-black hole (PSR-BH) systems are to be expected. Being timed with the SKA, a PSR-BH system would be an amazing probe of relativistic gravity with a discriminating power that surpasses all of its present and foreseeable competitors [16].

\subsection{Black Hole properties}

As stars rotate, astrophysicists also expect $\mathrm{BHs}$ to rotate, giving rise to both a $\mathrm{BH}$ spin and quadrupole moment. The resulting gravito-magnetic field causes a relativistic frame-dragging in 
the $\mathrm{BH}$ vicinity, leading the orbit of any test mass about the $\mathrm{BH}$ to precess if the orbit deviates from the equatorial plane. The consequences for timing a pulsar around a BH have been studied in detail by Wex \& Kopeikin [17], who showed that the study of the orbital dynamics allows us to use the orbiting pulsar to probe the properties of the rotating $\mathrm{BH}$. Not only can the mass of the $\mathrm{BH}$ be measured with very high accuracy, but the spin of the $\mathrm{BH}$ can also be determined precisely using the nonlinear-in-time, secular changes in the observable quantities due to relativistic spinorbit coupling. The anisotropic nature of the quadrupole moment of the external gravitational field will produce characteristic short-term periodicities due to classical spin-orbit coupling, every time the pulsar gets close to the oblate BH companion [18, 17]. Therefore, the mass, $M$, and both the dimensionless spin $\chi$ and quadrupole $q$,

$$
\chi \equiv \frac{c}{G} \frac{S}{M^{2}} \quad \text { and } \quad q=\frac{c^{4}}{G^{2}} \frac{Q}{M^{3}}
$$

of the BH can be determined, where $S$ is the angular momentum and $Q$ the quadrupole moment. These measured properties of a BH can be confronted with predictions of GR.

In GR, the curvature of space-time diverges at the centre of a $\mathrm{BH}$, producing a singularity, which physical behaviour is unknown. The Cosmic Censorship Conjecture was invoked by Penrose in 1969 (see e.g. [19]) to resolve the fundamental concern that if singularities could be seen from the rest of space-time, the resulting physics may be unpredictable. The Cosmic Censorship Conjecture proposes that singularities are always hidden within the event horizons of $\mathrm{BHs}$, so that they cannot be seen by a distant observer. A singularity that is found not to be hidden but "naked" would contradict this Cosmic Censorship. In other words, the complete gravitational collapse of a body always results in a $\mathrm{BH}$ rather than a naked singularity (e.g. [20]). We can test this conjecture by measuring the spin of a rotating BH: In GR we expect $\chi \leq 1$. If, however, SKA observations uncover a massive, compact object with $\chi>1$, two important conclusions may be drawn. Either we finally probe a region where GR is wrong, or we have discovered a collapsed object where the event horizon has vanished and where the singularity is exposed to the outside world.

One may expect a complicated relationship between the spin of the $\mathrm{BH}, \chi$, and its quadrupole moment, $q$. However, for a rotating Kerr BH in GR, both properties share a simple, fundamental relationship [21], i.e. $q=-\chi^{2}$. This equation reflects the "No-hair" theorem which implies that the external gravitational field of an astrophysical (uncharged) BH is fully determined by its mass and spin. Therefore, by determining $q$ and $\chi$ from timing measurements with the SKA, we can confront this fundamental prediction of GR for the very first time.

The best timing precision would be provided by a PSR-BH system with a MSP companion. Such systems do not evolve in standard scenarios, but they can be created in regions of high stellar density due to exchange interactions. Prime survey targets would therefore be the innermost regions of our Galaxy and Globular Clusters. Finding pulsars in orbits around massive or super-massive BHs would allow us to apply the same techniques for determining their properties as for the stellar counterpart [17]. Since the spin and quadrupole moment of a BH scale with its mass squared and mass cubed, respectively, relativistic effects would be measured much easier.

\subsection{Gravitational Wave Background}

The SKA will discover a dense array of MSPs distributed across the sky. Being timed to very high precision $(<100 \mathrm{~ns})$, this "Pulsar Timing Array" (PTA) acts as a cosmic gravitational wave 


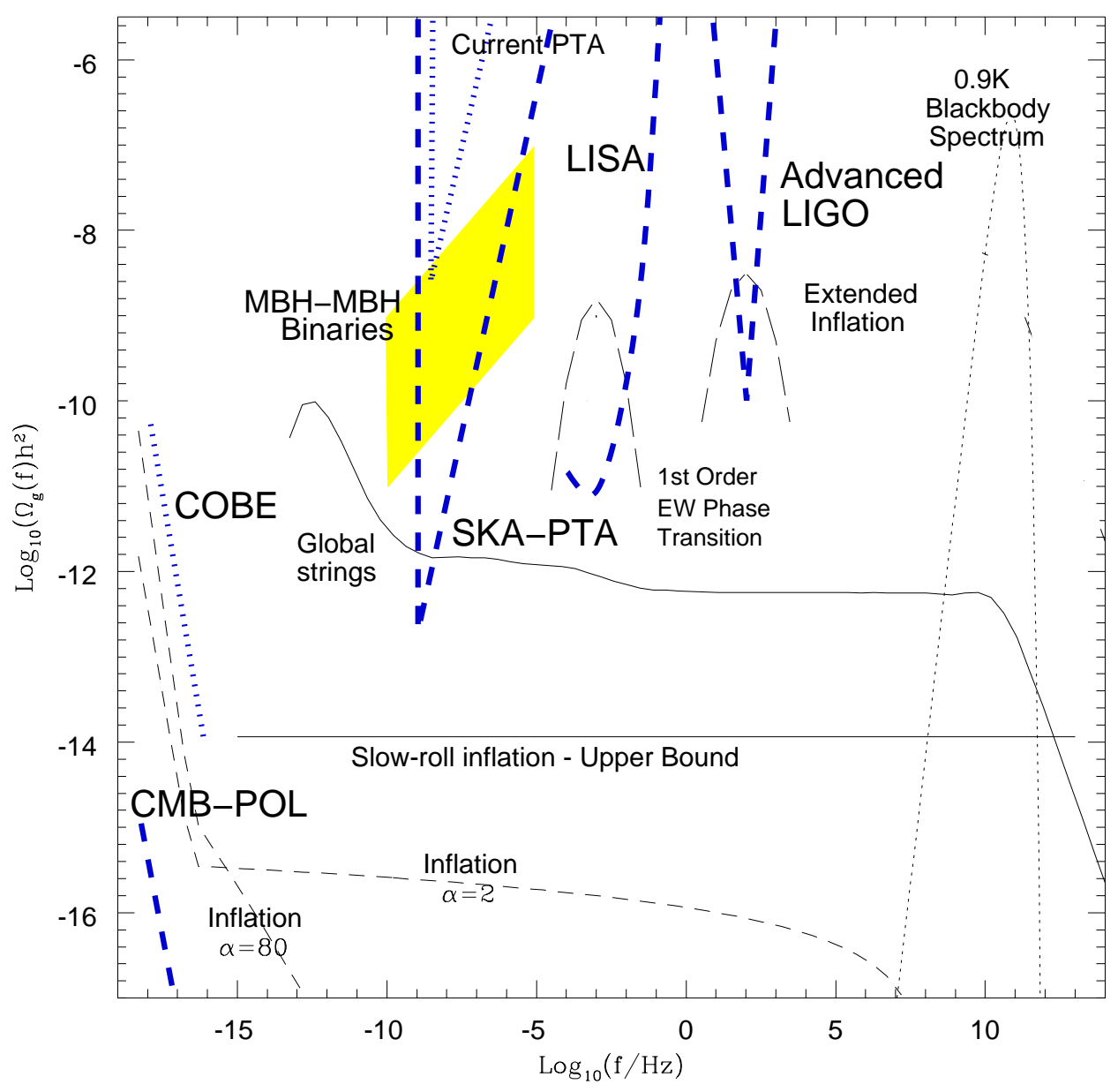

Figure 2: Summary of the potential cosmological sources of a stochastic gravitational background and sensitivity curves for various experiments (see [25, 14]).

(GW) detector. Each pulsar and the Earth can be considered as free masses whose positions respond to changes in the space-time metric. A passing gravitational wave perturbs the metric and hence affects the pulse travel time and the measured arrival time at Earth [22, 23, 24]. With observing times of a few years, pulsars are sensitive to GWs frequencies of $f>1 / T$, hence in the $\sim \mathrm{nHz}$ range. Consequently, the SKA can detect the signal of a stochastic background of GW emission in a frequency range that is complementary to that covered by LISA and LIGO.

A stochastic gravitational wave background should arise from a variety of sources. Cosmological sources include inflation, string cosmology, cosmic strings and phase transitions (see Figure 2). We can write the intensity of this GW background as

$$
\Omega_{\mathrm{gw}}(f)=\frac{1}{\rho_{c}} \frac{d \rho_{g w}}{d \log f}
$$

where $\rho_{\mathrm{gw}}$ is the energy density of the stochastic background and $\rho_{c}$ is the present value of the critical energy density for closure of the Universe, $\rho_{c}=3 H_{0}^{2} / 8 \pi G$ with $H_{0} \equiv h_{0} \times 100 \mathrm{~km} \mathrm{~s}^{-1}$ 
$\mathrm{Mpc}^{-1}$ as the Hubble constant. A contribution to the GW background is also expected from astrophysical processes, i.e. the coalescence of massive BH binaries during early galaxy evolution [24, 26]. The amplitude of this signal depends on the mass function of the massive BHs and their merger rate [26]. Measuring the slope of the spectrum would allow us to discriminate between this foreground signal and the cosmological sources. The wedge-like sensitivity curve of the PTA is shown in Fig. 2. For timing precision that is only limited by radiometer noise, the RMS is expected to scale with the collecting area of the observing telescope. In reality, the precision is also affected by other effects. Their limiting influence and the application of correction schemes will need to be determined on a case by case basis. However, extrapolating from the experience with the best performing MSPs today, we can expect the SKA to improve on the current limit on $h_{0}^{2} \Omega_{\mathrm{gw}}$ by a factor $\sim 10^{4}$ ! Further details can be found in the contribution by Jenet at this conference.

\section{Summary}

The applications of binary pulsars for the tests of theories of gravity are well known. Therefore, it is surprising that even 40 years after the discovery of pulsars, and more than 30 years after the discovery of the first binary pulsar, the exciting results obtained via the observations of pulsars do not seem to stop coming. That is the case even before the SKA will be switched on during the next decade. With the SKA, a complete new level of pulsar astrophysics will be enabled. Thousands of millisecond pulsars and hundreds of relativistic binaries will provide a large sample of compact systems which are superb probes for testing relativistic gravity. In particular, we can expect that pulsar - black hole systems will be found. With those it will be possible to study the properties of black holes, allowing us to compare them to predictions by general relativity and its Cosmic Censorship Conjecture and the No-Hair Theorem. The fun has only just begun.

\section{References}

[1] Burgay, M., D’Amico, N., Possenti, A., Manchester, R. N., Lyne, A. G., Joshi, B. C., McLaughlin, M. A., Kramer, M., Sarkissian, J. M., Camilo, F., Kalogera, V., Kim, C., \& Lorimer, D. R. 2003, Nature, 426, 531

[2] Lyne, A. G., Burgay, M., Kramer, M., Possenti, A., Manchester, R. N., Camilo, F., McLaughlin, M. A., Lorimer, D. R., D’Amico, N., Joshi, B. C., Reynolds, J., \& Freire, P. C. C. 2004, Science, 303, 1153

[3] Manchester, R. N., Lyne, A. G., Camilo, F., Bell, J. F., Kaspi, V. M., D’Amico, N., McKay, N. P. F., Crawford, F., Stairs, I. H., Possenti, A., Morris, D. J., \& Sheppard, D. C. 2001, MNRAS, 328, 17

[4] Kalogera, V., Kim, C., Lorimer, D. R., Burgay, M., D’Amico, N., Possenti, A., Manchester, R. N., Lyne, A. G., Joshi, B. C., McLaughlin, M. A., Kramer, M., Sarkissian, J. M., \& Camilo, F. 2004, ApJ, 601, L179

[5] Kramer, M., Stairs, I. H., Manchester, R. N., McLaughlin, M. A., Lyne, A. G., Ferdman, R. D., Burgay, M., Lorimer, D. R., Possenti, A., D’Amico, N., Sarkissian, J. M., Hobbs, G. B., Reynolds, J. E., Freire, P. C. C., \& Camilo, F. 2006, Science, 314, 97

[6] Kramer, M., Stairs, I. H., Manchester, R. N., McLaughlin, M. A., Lyne, A. G., Ferdman, R. D., Burgay, M., Lorimer, D. R., Possenti, A., D’Amico, N., Sarkissian, J. M., Reynolds, J. E., Joshi, B. C., Freire, P. C. C., \& Camilo, F. 2006, Annalen der Physik, 15, 34 
[7] Damour, T. \& Taylor, J. H. 1992, Phys. Rev. D, 45, 1840

[8] Weisberg, J. M. \& Taylor, J. H. 2005, in Binary Radio Pulsars, ed. F. Rasio \& I. H. Stairs (San Francisco: Astronomical Society of the Pacific), 25

[9] Stairs, I. H., Thorsett, S. E., Taylor, J. H., \& Wolszczan, A. 2002, ApJ, 581, 501

[10] Taylor, J. H., Wolszczan, A., Damour, T., \& Weisberg, J. M. 1992, Nature, 355, 132

[11] Wex, N. \& Kramer, M. 2007, MNRAS, 380, 455

[12] Damour, T. \& Deruelle, N. 1985, Ann. Inst. H. Poincaré (Physique Théorique), 43, 107

[13] Damour, T. \& Deruelle, N. 1986, Ann. Inst. H. Poincaré (Physique Théorique), 44, 263

[14] Kramer, M., Backer, D. C., Cordes, J. M., Lazio, T. J. W., Stappers, B. W., \& Johnston, S. . 2004, 48, 993

[15] Cordes, J. M., Kramer, M., Lazio, T. J. W., Stappers, B. W., Backer, D. C., \& Johnston, S. 2004, 48, 1413

[16] Damour, T. \& Esposito-Farèse, G. 1998, Phys. Rev. D, 58, 1

[17] Wex, N. \& Kopeikin, S. 1999, ApJ, 513, 388

[18] Wex, N. 1998, MNRAS, 298, 67

[19] Hawking, S. W. \& Penrose, R. 1970, Royal Society of London Proceedings Series A, 314, 529

[20] Wald, R. M. 1984, General relativity (Chicago: University of Chicago Press, 1984)

[21] Thorne, K. S., Price, R. H., \& Macdonald, D. A. 1986, Black Holes: The Membrane Paradigm (New Haven: Yale Univ. Press)

[22] Detweiler, S. 1979, ApJ, 234, 1100

[23] Foster, R. S. \& Backer, D. C. 1990, ApJ, 361, 300

[24] Rajagopal, M. \& Romani, R. W. 1995, ApJ, 446, 543

[25] Battye, R. A., Shellard, E. P. S., 1996, Classical Quantum Gravity, 13, 11A, A239

[26] Jaffe, A. H. \& Backer, D. C. 2003, ApJ, 583, 616 